\title{
FRACTAL - MATRIX METHOD FOR NEW PRODUCT DEVELOPMENT
}

\author{
Georgi Todorov \\ Technical University of Sofia, Faculty of Industrial Technology, \\ 8, St. Kliment Ohridski Blvd, 1756 Sofia, Bulgaria, \\ e-mails: gdt@tu-sofia.bg
}

\begin{abstract}
The paper is dedicated to innovative, fractal method of composing complex systems for rapid implementation of variants, modular re-use of components for high flexibility and efficiency of organization and management of products and processes throughout the life cycle of the products. The level of structuring is not limited and each level that is incorporated in the previous one has a fractal matrix relative to the system level. The new Fractal Matrix Method is worked out as having many input points and many possible outputs, depending on the project stage, the level of technological readiness and level of structuring of individual systems and fragmentation at a component level. The main advantage of the Fractal Method with simplistic self-similar structural solutions is that the process of development is much more relevant to actual process of composition of new products dominantly using modules or supplied components, allowing rapid development, modular approach, reusability of components, efficient change management, self-development in VP dimensioning and many other design and technological advantages, as well as the possibility of involving additional design and engineering resources to the different stages or levels of technology readiness and last but not least - easy tracking of the development process.

Keywords: Fractal Matrix Method, new product development, module approach, complex product design.
\end{abstract}

\section{STATE OF THE ART}

The need for sustainable development, combined with the continuous improvement of the competitiveness is mandatory and only one way to provide it is a continuous innovation process and the introduction of new products, technologies and methods of organization. In the process of designing it is necessary to solve the problem of choosing the optimal (rational, effective by

DOI: $10.7546 /$ EngSci.LVII.20.01.05

Engineering Sciences, LVII, 2020, No. 1 
different criteria) variant repeatedly. The choice of evaluation criteria is also crucial for the proper conduct of the whole process and especially for the ultimate success.

In most modern design methods [1], an iterative process is recommended, in which the possibility of returning to the previous step is not forbidden, but on the contrary, is encouraged in order to adjust and complete the model. A number of methods and approaches are devoted to the design organization. One of the most widely adopted approaches is the German Society of Mechanical Engineers' VDI 2221 standard and provides guidance on a systematic approach for the development and construction of technical systems and products [2].

The goal of these guidelines is to give a common methodology for the design of technical systems and products, and to support methodologically and systematically the designers in order to create efficient way of working. The guidelines are independent of the content of the technical field and the organizational and design aspects. One major aspect is the integrated processing of 3D models, based on the application of CAD systems [3]. The sequence of building steps should not be considered invariable - some steps may be skipped and others often repeated. This flexibility is in line with the practical application of the design process and is very important for the actual implementation of all design methods [4]. Particular emphasis is placed on the iterative nature of the process.

A design method that clearly demonstrates the iterative nature of the process and the need to repeatedly solve the problem of finding the optimal structural variant on the example of automation systems is the Boyadzhiev method [5]. The Ulrich method [4, 6], Simpson [7], also provides for the iterative execution at separate stages of the design process. In [8], a typical design algorithm is proposed that consists of three main steps: synthesis, analysis, and optimization, which are consistently performed. Therefore, one of the most responsible steps in the design process is the stage at which the structure is created, i.e. the types and relationships between the structure components are determined $[3,5,9]$. This stage is very important as it serves as the basis for further design, and the correction of an wrongly chosen solution at a later stage will provoke significant costs.

The analysis of the design practices shows the development from individual thinking and action towards a holistic, general and corporately oriented direction, which is an essential element of this methodology. Designers need to have a holistic view of the design process in order to have an adequate understanding of the purpose and seek to apply it appropriately. The review shows 
the need of adopting effective new design methods and approaches relevant to very fast changing new complex and module based design architectures.

The objective of this research is the development of a new integral approach to functional-structuring and level of technology readiness in the stage of the product development.

\section{MOTIVATION}

Science, industry and research must work more closely to bridge the gap between the design methods offered by science and the methods required by industry. In order to gain experience, science must go into industry and adopt its methods, gathering impressions of good practices in industry [9, 10, 11]. Related to VDI 2221, this would mean generating a further description of the guidelines adapted to the specifics of the individual project, as well as incorporating the identified problems and their solutions. This description should include an indication of the most important facts about the work (thinking and action) of the designer already mentioned in the VDI guidelines. At the same time, it is noticed that the increasing variety of products and the decrease of the volumes produced from each specific model lead to the emergence of modular platforms and variant systems, different configurations, specific performances for different markets and many others, which makes the products multifarious and branched structures.

On the other hand, the life of the products becomes shorter over time, which requires they to be updated at least partially after a short period of time due to market requirements or regulatory reasons.

Third, the approaches and organization that are increasingly delegated in the practice of new product development are delegated to individual stages of product development or the overall development of individual sub-units or subcontractors. This is a sustainable trend in work models through division of labor, high levels of specialization and levels of competence, as well as globalization processes.

In this respect, the successive iterative development methods and approaches discussed above are not fully adequate and effective as design organization methods and technology for modern highly structured horizontal (using unified components or modules), vertical (individual components evolving and supply from a large number of subcontractors) and accelerated processes of development, especially when creating more complex products with large Roeqtans.

Engineering Sciences, LVII, 2020, No. 1 
Development processes are increasingly fragmented, and very often fragmentation is performed at the functional level - elements, units or modules with clearly defined interfaces are distinguished, as well the different stages and levels of technological readiness in the development process. One of the approaches to designing complex systems is the so-called "V" approach (VDI 2206) to decomposing and subsequent integrating into the design process [12, 13]. However, it does not take into account the interplay between individual units at complex systems, such as modular or unified systems, which is not effective in multi-variant construction.

To a large extent, the way of organizing the project activity in complex engineering projects with full or partial design cycle, with more participants in the different stages and taking into account the mutual influence of the individual units and elements of the product, updated the "W" approach [14, $15]$ is used to train designers in new design methods and adapt them to specific needs is critical important $[16,17]$.

This is even more so for modular systems, where it is not possible and necessary to prototype all modules and all combinations of modules.

\section{THE NEW METHOD DEVELOPMENT}

In order to avoid the disadvantages of "V", as well as for various more complex cases of "W" approach, a new Fractal Approach for the New Product Development Process (PDP) is worked out.

A fractal approach to systems development has been applied in a number of areas $[18,19]$. Theoretically, fractal systems are self-organizing, cooperative, and self-similar at different levels of systems that can adapt to changing system goals and working environment. The resilience of such systems are the attractive features in the organization of development processes in a rapidly changing technological environment.

The systems are building as scaling up and reusing of the subsystems (fractals), Fig. 1. In the fractal approach, the characteristic of the structure is identical to the fragmentation levels, either in terms of complexity or variability, where the highest level relates to the whole product and the lowest to the smallest element/node. Using the fractal properties over the general approach from a process perspective, "self-similarity" feature can be viewed in three different layers, namely:

- Structure of the elements - for example, a separate unit or "Module" (structure product elements);

- Functionality - functionally separated systems (drives, sensors); 

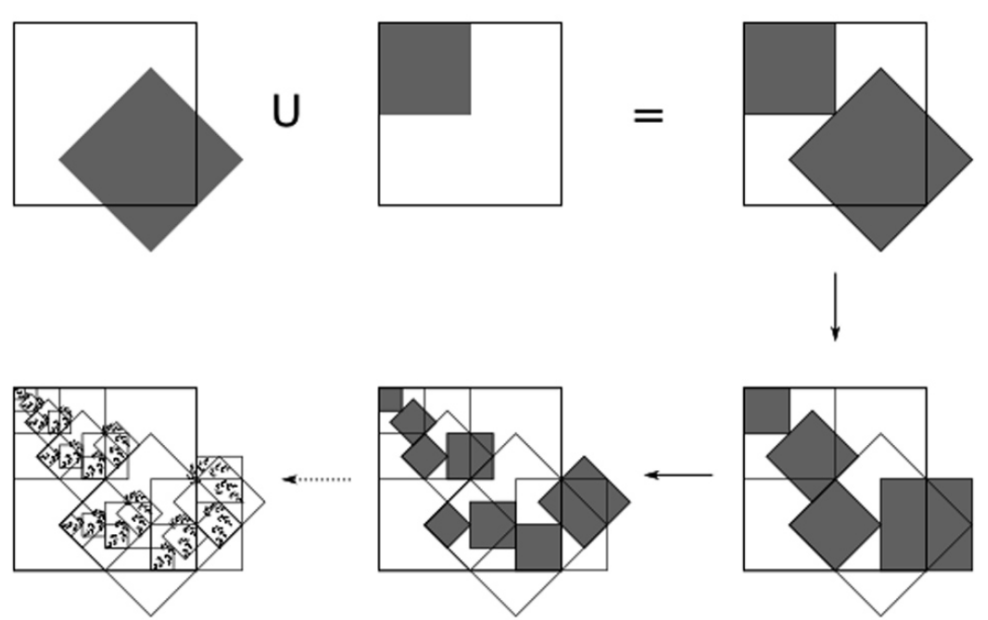

Fig. 1. Fractals generation by square shape multiplication - square parameters are: $p 1=1+12 i$, $p 2=i,=1=\sqrt{12} e \pi 4 i,=2=12 e 0 i$ (Jozsef, 2013)

- Information architecture.

The characteristic properties of the fractal principle of organization can be summarized as follows [11]:

- The fractal method is based on the self-organization of functions or activities based on the similarity;

- Self-improvement - fractals self-adjust based on the feedback, i.e. optimize their shape and behaviour (geometric, energy, information, interface, etc.), allowing them to adapt to any change of environment that exists (contextual response to external pressure);

- Sustainability: their interaction with the environment (temporal and spatial evolution) is accomplished by generating and integrating the necessary diversity to achieve the goal - proper placement in the structure;

- Fractally based systems are opened - share feedback with the environment;

- Fractals are behaviorally nonlinear systems - their interaction with the environment and their sustainability are based on radical adaptation and gradual innovation [4];

- A set of features that allows them to adapt to technical requirements have been determined.

Therefore, they allow the analogy and the process of transposition by fractal units (virtual or physical objects) to be established so that a sustainable development of the structure can be achieved in its composition or expansion. 
These properties are particularly important in the need to repeatedly apply identical or similar, new related processes in the development or adaptation of complex systems.

The basic idea behind this approach is to apply similar "fractal" elements to the development process, in the presence of a large number of variants or surnames made up of unified elements or even more modularly built. In this sense, it is appropriate the principle of modular construction to base on a fractal approach. Applicability comes in two main forms:

- Designing bottom-up structures - When generating variants or generalized rows of nodes and elements, it is necessary to apply identical "fractal" approaches and design steps, for example, to use identical or fractal design approaches and steps to developing the product design and the development steps, structurally similar elements with similar parameters.

- Prototyping and validating behaviour - In variant or modular construction, a large number of variants or client implementations can be created. The main question for validating a particular variant or personalized solution in this case is how to prototype and validate each variant in order to reduce the risk of the effects of the interplay of untested variant compositions, without tests of each composition variant.

There are two approaches to this activity in practice:

- By analogy with the closest such composition with known behaviour;

- Based on the known behaviour of the individual elements/modules, the behaviour of the whole composition is assumed.

In complex systems with strong interconnectedness and interplay of the elements, both approaches are risky and do not allow the compositional effects to be evaluated.

Taking into account the shortcomings of the existing approaches to prototyping composite variants described above, a new approach for sustainable fractal design is proposed and defined here, which is applicable to the design of complex type systems, and in particular by familization or personalizaton of a product that meets customer requirements as best as possible through the compositional application of the fractal approach.

The approach is based on the deterministic compositional design for variants development by configuring modules or variant configuring metrics for the identity of the entire compositing, prototyping and validation tools.

This includes the requirements of the fractal design model - to create a fractal architecture for the steps of each at the stages of project development, structuring and defining them so that they can be used as sustainable elements for the level of each element or module independently of interfaces differences. 
The proposed approach is not closed and future research will be expanded to different levels of development of complex systems, especially as regards the virtual prototyping of complex composite objects at the stage of their development.

As mentioned above, complex modular systems do not allow complete testing, even with a virtual prototype of all compositional variants and solutions up to the level of personalization, due to the very large number of implementations. The fractal approach offers the possibility of composing a fractally constructed VP that is adequate to the physical composition of the product.

This work also develops the way of processing the information level of formation of 3D models and the associated levels of VP. Several basic principles of fractal theory can be outlined:

- Principle of fractal connectivity and organization - This principle establishes the requirements for organizing all procedures, such as deterministic fractal processes, for adapting to the chaotic dynamics of changing requirements, Fig. 2. This principle defines a set of organizational elements of the fractal approach in order to provide a system with self-similarity (a system whose elements are characterized by the dynamism and sustainability of the type of elements and the relationships between them), guidelines for self-organization when requirements change. This allows adaptating to the environment through the self-organization of fractal design processes (such as open and process-based and recursively defined innovative activities). They need to be stable in their development - this will be possible thanks to a set of parameters (metrics) and criteria that serve as an active and proactive way of adapting to the dynamics of customer requirements (internal restructuring of features as needed)
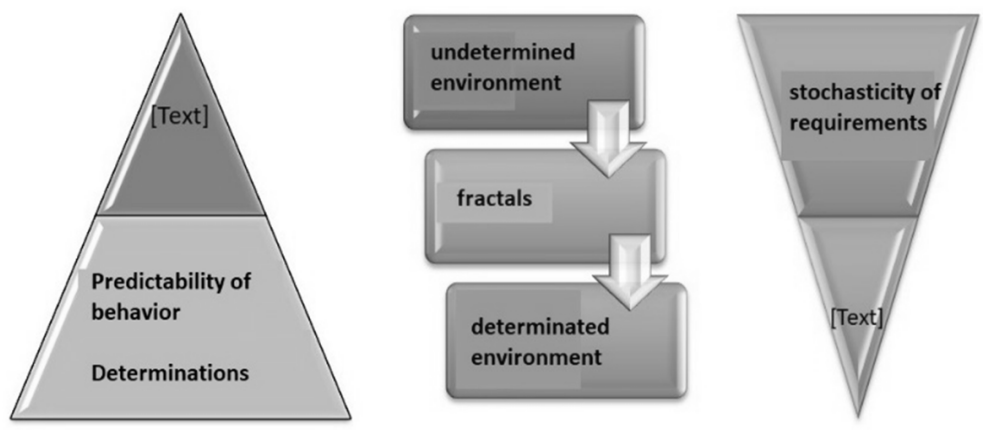

Fig. 2. Determinism and openness. Fractal approach to design 


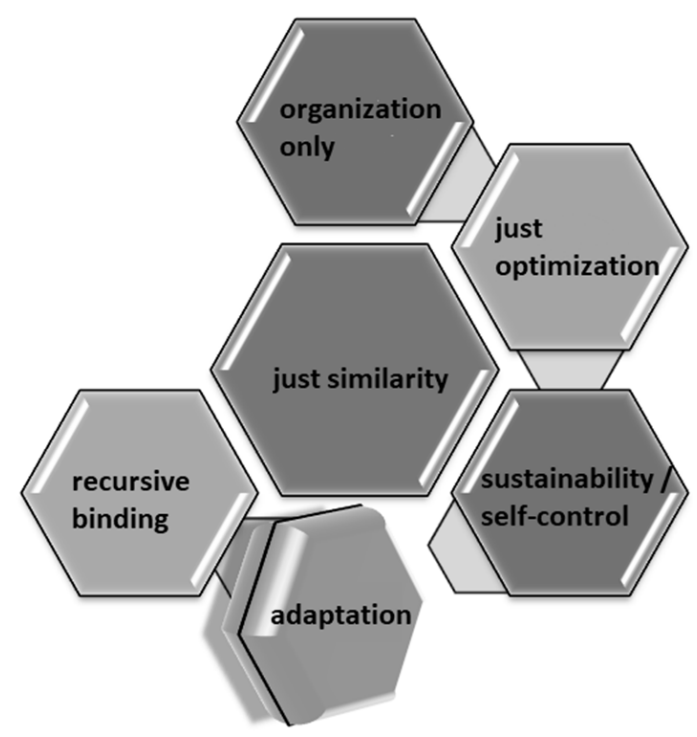

Fig. 3. Principles of fractal design approach

- The principle of fractalisation of the way of designing a sustainable product This principle develops the relationship between the fractal structure of the design organization and the market environment in order to achieve sustainable behaviour throughout the product life cycle. This principle involves integrating the technical, economic and environmental perspectives of fractal design lifecycle approaches in terms of adaptation behaviour required (minimal complexity of retrofit caused or recycling costs). The fractal design approach must be so organized and configured in recursive forms that the system complexity to be minimized and its stability assured, Fig. 3.

- Principle of fractal levels in design - This principle determines the level of fractality. The fractal design approach will be structured at different fractal levels in order to move and achieve rapid adaptation of the modification process or "template" order similar to a natural fractal object. A hierarchical structure of: (i) macro level (which integrates product specification), (ii) meso level (conceptual build, product functionality), (iii) micro level (customer order, core modules, additional interfaces) has been drawn up, and even at the "nano" level when designing a fractal architecture of a particular individual finite indivisible object (detail) - Fig. 4.

The last level of fractal architecture for component construction (especially inside the details) of artificial bone type follows the bionic way of forming component architecture or so-called "bio" design. These innovative forms of 

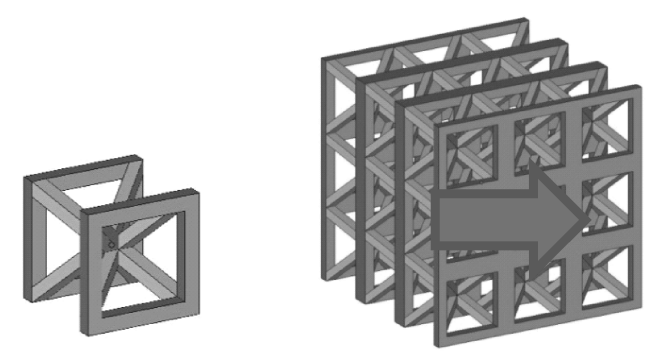

(a)
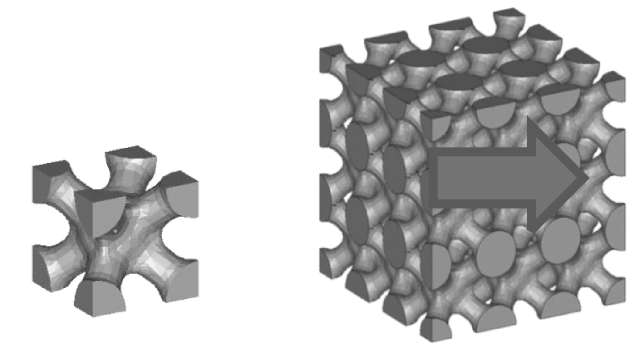

(b)
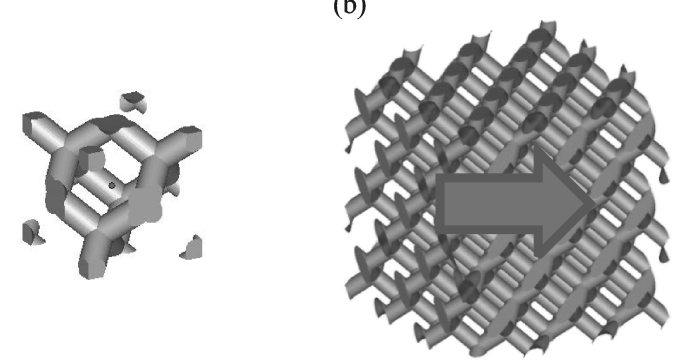

(c)

Fig. 4. Fractal levels in design - nano level of fractal architecture of a specific individual object (component) and virtual fragment based on fractal elements: (a) square with main diagonals; (b) diagonal structure with nodes;

(c) diamond like structure

fractal architecture in their geometry are difficult to produce with conventional manufacturing technologies, but are well suited to using additive methods and open up new horizons for designers of complex objects (details) with unknown in terms of weight and strength and enormous potential in critical structures. Three fractal shaped structures are shown in Fig. 4, on the left, the fractal on which they are based and constructed by "cloning" this fractal into larger structures is shown. 
Level separation provides the three-dimensional design system with the self-similarity and dynamics of managing each level or scaling in depth (recursive nesting) as needed. This structure reduces complexity, guarantees the resilience of the system and allows for self-organization, self-regulation and self-control without the need for corrective action and efficiency (without intervention, unnecessary waste of resources or duplication of information).

In the product concept development phase, the project work is multidisciplinary, but limited to a small group of highly qualified professionals. This is the step when creating the product structure, including when it comes to defining interfaces. In complex systems, the product structure has many levels, ranging from general schematics and top-level work frames to separate standalone components.

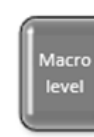

Product specification
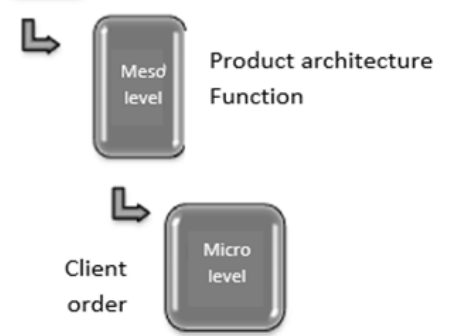

ㄴ

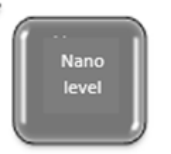

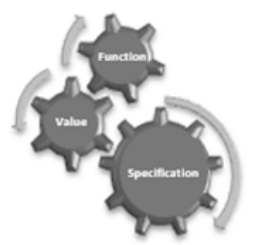

M1
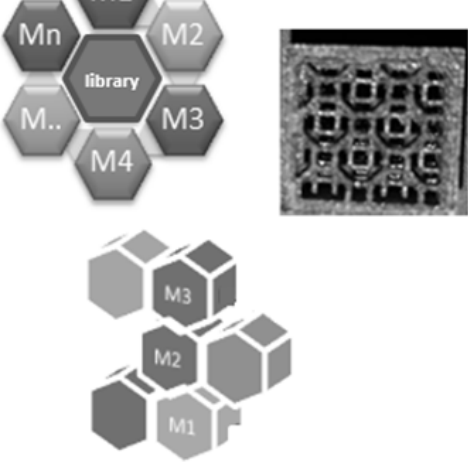

凹
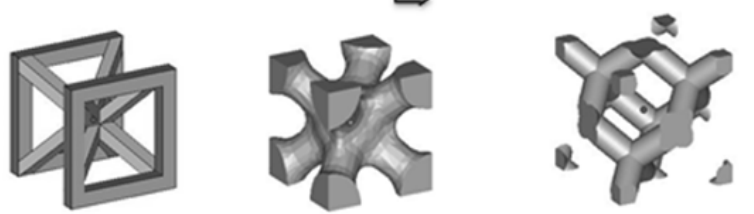

Fig. 5. Fractal levels in design - from fractal architecture to customer order and virtual prototype based on fractal elements 
At each level, there are specifications and interface definitions that are absolutely necessary to separate development activities, Fig. 5.

The levels of product structuring of nodes and elements are in the third dimension (in depth). The method is matrix-wide at the system (product) level, and is fractal [19] in structuring the system of levels [16, 18] and fragmenting each of the levels (sub-elements). Each level has its own subsystem matrix, similar to the system (fractal to it). In this method, the strategy for solving problems related to the interaction of individual units and elements of the product under operational conditions is defined in two possible approaches:

- The first approach is to maximize the separation of structural units with a precise definition of their input-output parameters and requirements (interface) to the work environment - a bottom-up approach. In this fragmentary structuring, each node (level) is developed, tested and implemented as fractal (autonomously, by an identical algorithm), after the product is evaluated at the system level - in the common matrix. This fractal approach gives a great deal of flexibility to the overall organization of the process and wide possibility for variant conception [20] and design, as well as for reuse of nodes and elements in other solutions. The disadvantage is that it is necessary to define in advance relatively precisely the interface parameters at each level, which in the initial stages of conception is difficult to achieve in its entirety.

- The second approach is to work with maximum consideration of the interplay of individual units and product elements at the system level, and to reach the desired indicators through iterative steps at the system level - the topdown approach. This approach involves less initial effort in structuring and fragmenting the entire system and produces faster results in simpler systems, but in development options requires a much larger resource for iterations aiming to take into account the interplay.

Composing modules for larger units allows descriptions to move up with the same level of abstraction. This reduces perceived complexity because the level of abstraction, i.e. the level of encapsulation of functional units in larger building blocks creates a simpler interface with other elements precisely by applying the Fractal Matrix Design Method (FMDM).

\section{CASE STUDY}

An example of Liftruck Family Development Project from the author's practice is used to illustrate modulization using the second approach - FMDM. Figure 6 shows the architecture of the systems [21] including chassi, cabine, mast, drivetrain, etc. 


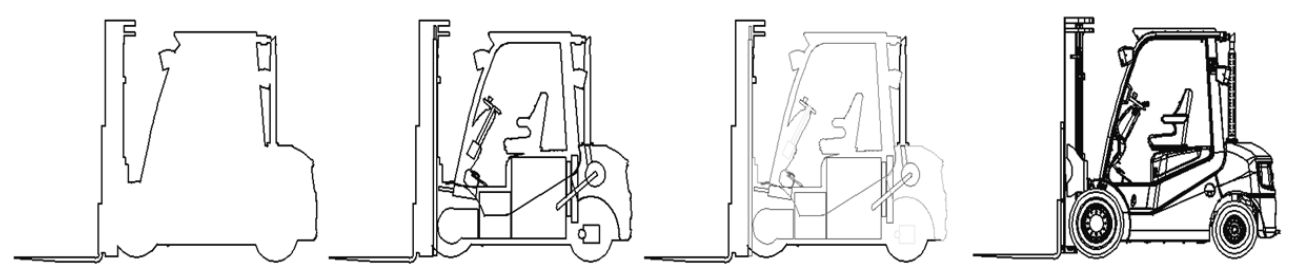

Fig. 6. System architecture of Liftruck FD25: left - system structure at system level showing the different machine systems and the relationships between them; right - a module-based structure represents a machine made up of modules and relationships between systems and modules

The modules consist of elements belonging to different systems, i.e. they have been developed by different engineering teams using FMDM.

Therefore, structural definitions through the FMDM are important both for integrating systems into modules and about the interfaces defined for each module, while simultaneously connecting the modules as interface and also separating them.

Modular interfaces can also include layers containing specific systems and their interfaces, as well as interfaces for exchanging data or flows to other systems. Figure 7 illustrates generally this way of using modular design as

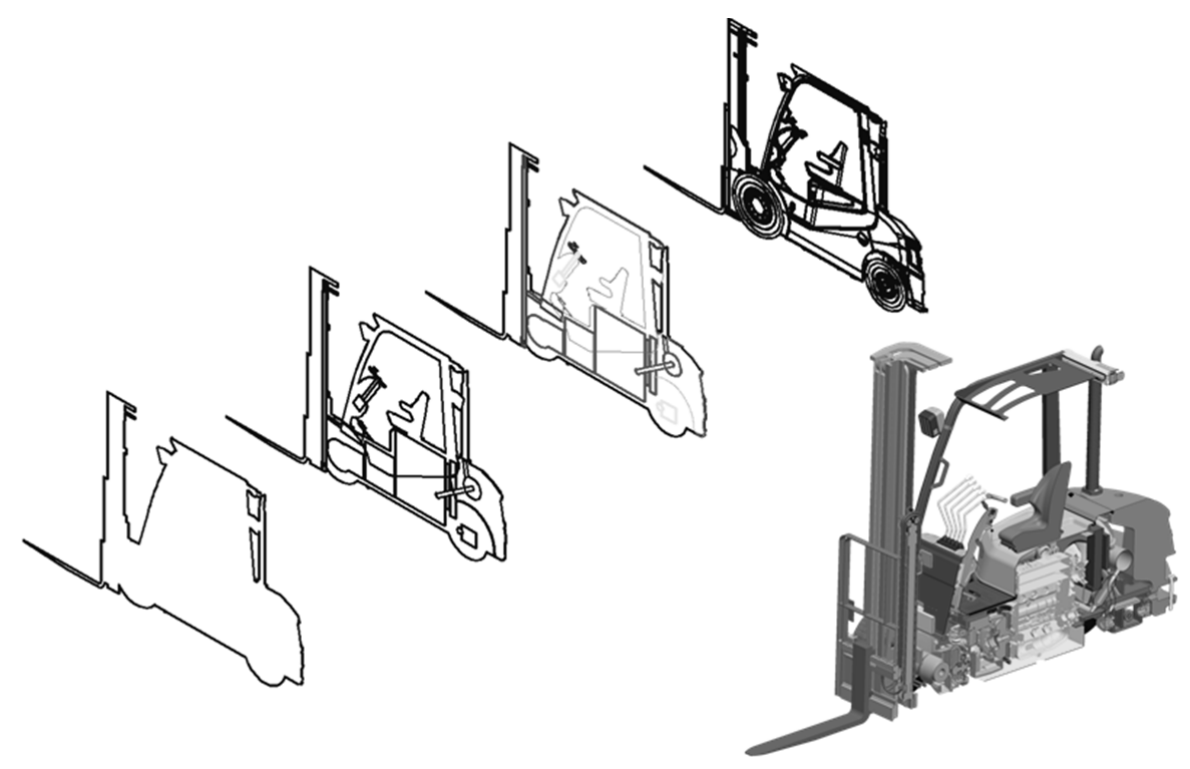

Fig. 7. Demonstration of different levels to structuring a product through FMDM 
an interface link structure for storing configuration-level storage across multiple product variants. The functionality of using modular structures, defined by their interfaces and geometry through the FMDM, also makes it possible to link data from CAD models to structural specifications. CAD structures can also be compiled and automated based on rules through the FMDM for connection in the context of modular construction with specific configuration parameters and functions.

In this methodology, there is a significant difference from system design based on the separation of individual systems, for example, hydraulic system, steering system, transmission, etc. Functional module separation is used here, each of which may include elements from different systems, for example, a chassis module includes two tanks - one for hydraulic fluid and one for fuel, which in system design should be to the hydraulic system and to engine/fuel supply, respectively.

The FMDM has the following advantages:

- Reducing the volume of project work;

- Reducing the cost of machinery due to the smaller number of different assemblies and parts that make them;

- Reducing design and production time by incorporating already installed modules - this is very important for custom-made unique machines;

- Increased reliability of machines;

- The reduced number of parts and assemblies facilitates machine maintenance and repair, etc.

\section{DISCUSSION}

Once the concept has been built on the product architecture, it is necessary to structure each subsystem, unit or component into a group or module that will have technical, financial and time goals. It is here that the field of application of the fractal recursively similar (self-similar) approach is discovered. Table 1 provides an expert evaluation of the applicability of the $\mathrm{V}, \mathrm{W}$ and Fractal approaches at different levels of complexity (along the abscissa axis) and variability (ordinate axis) of the product, such as structure and composition at the compositional level. Hence, it can be seen that the area of applicability of the FMDM demonstrated itself in complex variant or modular based product architectures with a high level of unification or personalization.

A further development of the fractal approach is in the stages of the objects of design that to be linked to the degree of technological maturity (feasibility,

Engineering Sciences, LVII, 2020, No. 1 
Table 1. Applicability of FMDM

\begin{tabular}{|c|c|c|c|c|}
\hline \multicolumn{2}{|c|}{} & \multicolumn{3}{|c|}{ Complexity } \\
\cline { 4 - 5 } & Typical & Variance & Modularity \\
\hline \multirow{3}{*}{$\begin{array}{c}\text { Degree of } \\
\text { Individualization }\end{array}$} & Configurations & "V" & "W" & "W" \\
\cline { 2 - 5 } & Variation & "W" & "W" & "W" / "Fractal" \\
\cline { 4 - 5 } & & & "Fractal" & "Fractal" \\
\cline { 4 - 5 } & Personalization & & "Fractal" & "Fractal" \\
\hline
\end{tabular}

material selection, production technology selection, compatibility with safety requirements).

Introduced FMDM gives a great deal of flexibility to the overall organization of the process and great possibilities for variants conception and design, as well as for reuse of nodes and elements in other solutions.

The disadvantage is that it is necessary to define in advance relatively precisely the interface parameters at each level, which in the initial stages of conception is difficult to achieve in its entirety.

\section{CONCLUSIONS}

- An innovative Fractal Matrix Method of composing the complex systems for rapid implementation of variants, modular re-use of components for high flexibility and efficiency of product and process organization throughout the life cycle of the product is worked out.

- The level of structuring is unlimited and each level that is incorporated in the previous one has a similar cloning fractal matrix relative to the system level.

- The main advantage of the fractal method, unlike currently used, is the possibility that the process of development is not considered and managed as iterative, but as having many input points and with many possible outputs, depending on the project stage, the level of technological readiness and level of structuring of each individual systems and fragmentation at a component level, using simplistic self-similar structural solutions.

- The developed FMDM allows rapid development, modular based design, reusability of components, efficient change management, self-development in VP environment and many other constructive and technological advantages on the one hand, as well as the openness of the development process with the possibility to attract additional design and engineering resources to the different stages or levels of readiness of the project. 


\section{ACKNOWLEDGEMENTS}

This work was supported by the European Regional Development Fund within the Operational Programme "Science and Education for Smart Growth 2014-2020" under the Project CoE "National Centre of Mechatronics and Clean Technologies" BG05M2OP001-1.001-0008.

\section{REFERENCES}

[1] S. Gladstone, A Fractal Approach to Innovation and Product Management, https://www.slideshare.net/SteveGladstone1/ a-fractal-approach-to-innovation-and-product-management

[2] VDI 2221, Methodik zum Entwickeln und Konstruieren Technischer Systeme und Produkte, VDI Vela (1993).

[3] G. Pahl, W. Beitz, J. Feldhusen, and K. H. Grote, Engineering Design: A Systematic Approach, Springer-Verlag, London (2007).

[4] K. T. Ulrich and S. D. Eppinger, Product Design and Development, McGraw-Hill (2008).

[5] I. Boyadjiev, B. Boyadjiev, and I. Malakov, Rechnerunterstuetzte Systemvorgehenweise Zur Variantenauswahl Beim Konstruieren, TU Vienna, Austria (1996).

[6] K. Ulrich And K. Tung, Fundaments of Product Modularity, in: Mechanical Design International, 1991, pp. 73-79.

[7] T. Simpson, J. Maier and F. Mistree, Product Platform Design: Method and Application, Research in Engineering Design (2001) 13 (1) 2-22.

[8] A. A. Shekhonin, B. M. Domnenko, and O. A. Gavrilina, Optical Instrument Design Methodology, $1^{\text {st }}$ Edition, ITMO, St. Petersburg (2006) (in Russian).

[9] H. Birkhofer, J. Jänsch, And H. Kloberdanz, An Extensive and Detailed View of the Application of Design Methods and Methodology in Industry, in: ICED 2005 Proceedings.

[10] S. D. Eppinger And T. R. Browning, Design Structure Matrix Methods and Applications, Cambridge, MA: MIT Press (2012).

[11] Peraltaa and Marcosb, Sustainable Fractal Manufacturing: A New Approach to Sustainability in Machining processes, https://doi.org/10.1016/j. proeng. 2015.12.579

[12] VDI 2206, Entwicklungsmethodik für Mechatronische Systeme (Design Methodology for Mechatronic Systems) (2004).

[13] K. Kamberov, M. Semkov, and B. Zlatev, Design Considerations through Study of Thermal Behaviour of Smart Poles, LNICST (2019) 283 327-338.

[14] G. D. Todorov and K. X. Kamberov, Virtual Engineering, Direct Services Ltd, Sofia (2015) (in Bulgarian). 
[15] The "W-Modell" - A Systems Engineering Based Approach to Active Systems Development, https://www.plmportal.org/en/research-detail/ the-w-modell-a-systems-engineering-based-approach-to-activesystems-development.html

[16] G. Todorov, Y. Lai, And K. Kamberov, Development of Collaborative Approach for Virtual Prototyping Based on 3D, in: MTM Congress Proceedings, Sofia, 2011, pp. 172-177.

[17] J. Ovtcharova, Virtual Engineering: Principles, Methods and Applications, in: International Design Conference - Design, Dubrovnik, 2010.

[18] V. JozSEF, On the Geometry of IFS Fractals and its Applications, a Ph. D. Thesis, Ontario: University of Waterloo (2013).

[19] P. Tromboni De Souza Nascimento and E. Pinheiro, The Fractal Structure for Integrated Product Development: A New Metaphor Based on the Case of EMBRAER. Salvador, Brasil, Encontro da ANPAD (2006).

[20] K. Fujita And H. Yoshida, Product Variety Optimization Simultaneously Designing Module Combination and Module Attributes, Concurrent Engineering: Research and Application (2004) 105-118.

[21] V. Nikolov, Mechanical Mathematical Modeling of the Tilting Process of Lifting Masts of Forklift Trucks with Load, Journal of the Technical University of Sofia, Plovdiv Branch, Fundamental Sciences and Applications, Plovdiv (2013) 19 (2) 347-350, ISSN 1310-8271.

Received March 29, 2020 